\title{
Socioeconomic inequalities in resilience and vulnerability amongst older adults: A population-based birth cohort analysis
}

\begin{tabular}{|r|l|}
\hline Journal: & International Psychogeriatrics \\
\hline Manuscript ID & IPG-06-17-230.R1 \\
\hline Manuscript Type: & Original Research Article \\
\hline Complete List of Authors: & $\begin{array}{l}\text { Cosco, Theodore; MRC Unit for Lifelong Health and Ageing; University of } \\
\text { Oxford, Oxford Institute of Population Ageing } \\
\text { Cooper, Rachel; MRC Unit for Lifelong Health and Ageing } \\
\text { Kuh, Diana; MRC Unit for Lifelong Health and Ageing } \\
\text { Stafford, Mai; MRC Unit for Lifelong Health and Ageing }\end{array}$ \\
\hline Keywords: & Resilience, Social support, Physical activity \\
\hline &
\end{tabular}

\section{SCHOLARONE ${ }^{m}$ \\ Manuscripts}


Socioeconomic inequalities in resilience and vulnerability amongst older adults: A population-based birth cohort analysis

Authors:

Cosco TD ${ }^{1,2}, \mathrm{PhD}$; Cooper $\mathrm{R}^{1}, \mathrm{PhD} ;$ Kuh $\mathrm{D}^{1}, \mathrm{PhD}$; Stafford $\mathrm{M}^{1}, \mathrm{PhD}$

${ }^{1}$ MRC Unit for Lifelong Health and Ageing at UCL, 33 Bedford Place, London WC1B $5 J U$

${ }^{2}$ Oxford Institute of Population Ageing, University of Oxford, 66 Banbury Road, Oxford, UK, OX2 6PR

Corresponding author: Theodore D Cosco, MRC Unit for Lifelong Health and Ageing at UCL, 33 Bedford Place, London WC1B 5JU; t.cosco@ucl.ac.uk 


\section{Abstract \\ Background:}

Ageing is associated with declines in physical capability; however, some individuals demonstrate high wellbeing despite this decline, i.e. they are "resilient". We examined socioeconomic position (SEP) and resilience and the influence of potentially modifiable behavioural resources, i.e. social support and leisure time physical activity (LTPA), on these relationships.

Methods:

Data came from the Medical Research Council National Survey of Health and Development, a nationally-representative birth cohort study. Resilience-vulnerability at age 60-64 ( $n=1756)$ was operationalised as the difference between observed and expected levels of wellbeing, captured by the Warwick-Edinburgh Mental Well-being Scale (WEMWBS), given the level of performance-based physical capability. SEP was assessed by father's and own social class, parental education, and intergenerational social mobility. PA and structural/functional social support were reported at ages 53 and 60-64. Path analysis was used to examine mediation of SEP and resilience-vulnerability through LTPA and social support.

Results:

Participants in the highest social class had scores on the resilience to vulnerability continuum which were an average of 2.3 units $(\beta=0.46,95 \% \mathrm{Cl} 0.17,0.75)$ higher than those in the lowest social class. Greater PA $(\beta=0.58,95 \% \mathrm{Cl} 0.31,0.85)$ and social support $(\beta=3.27,95 \% \mathrm{Cl} 2.90,3.63)$ were associated with greater resilience; LTPA partly mediated participant social class and resilience (23.4\% of variance). 
Conclusions:

Adult socioeconomic advantage was associated with greater resilience. Initiatives to increase LTPA may contribute to reducing socioeconomic inequalities in this form of resilience in later life.

Keywords: resilience, social support, physical activity, socioeconomic position 


\section{INTRODUCTION}

As the number of older adults increases worldwide, understanding the relationship between physical and psychological health is increasingly important. It is well established that the likelihood of physical limitations and multimorbidity increase with age (Manton et al., 1997; Wister et al., 2016). Although lower levels of physical capability, that is the capacity to undertake the physical tasks of daily living (Cooper et al., 2014a), have been associated with lower levels of mental wellbeing (Cooper et al., 2014b), individuals in their 60s and 70s may also demonstrate high levels of mental wellbeing, a phenomenon often referred to as the "wellbeing paradox" (Windle et al., 2010), or "disability paradox" (Albrecht and Devlieger, 1999). These findings suggest that individuals' emotional responses to poor physical capability vary from positive to negative (Antonovsky, 1996) and that responses exist on a continuum of resilience, i.e. having higher levels of wellbeing than would be expected given the level of physical capability, to vulnerability, i.e. having lower levels of wellbeing than would be expected given the level of physical capability.

Research into resilience and vulnerability has its roots in developmental psychology, examining how certain children managed to fare better than others despite experiencing similar levels of adversity (Masten, 2001). These studies of early life have subsequently been expanded to later life (Ong et al., 2006), examining the ways in which older adults respond more positively to a circumstance than would be anticipated based on the level of adversity experienced. Previous methodological work by Miller-Lewis, et al. (2013) suggests that this conceptual framework can be used to operationalise a continuum of resilience to vulnerability via residual values from linear regression models. This approach quantifies the difference between the observed and expected, or fitted, values. To help understand the disability paradox, 
it can be used to quantify how much higher (or lower) than expected is a person's mental wellbeing than would be expected given their observed level of physical capability. This salutogenic perspective on the ageing process draws attention to processes distinct from pathogenic and disease-oriented pathways (Wister et al., 2016).

There are two possible theories that could explain the development of resilience. First, the ability of ageing individuals to be resilient is hypothesised to be related to the resources available to them; specifically social, individual, and environmental resources (Wister et al., 2016). The 'life course model of multimorbidity resilience' theorises that individuals who build up reserves, by invoking these three resource domains, are better able to accommodate adversity (Wister et al., 2016). Further, this model suggests a cumulative approach to the development of resilience, i.e. over time reserves are developed in these resource areas in order to foster more positive responses to adversity during the ageing process. This suggests that individuals who have higher levels of these resources currently at their disposal will have greater resilience; therefore, we hypothesise that individuals of higher socioeconomic position (SEP) will have greater resilience, as they have greater social and economic resources available to them when compared to individuals of lower SEP. It is well established that health is socioeconomically pattern (Marmot, 2005). Those in socioeconomically disadvantaged groups have been shown to have greater risk of a host of negative physical and psychological outcomes when compared with socioeconomically advantaged groups (Marmot, 2005). Less clear, however, is whether greater socioeconomic advantage in childhood as well as adulthood yields resources that promote resilience. The second theory proposes a different association between childhood SEP and later life resilience. In contrast to 
the 'life course model of multimorbidity resilience', the theory of 'steeling' suggests that resilience may be developed through inoculation by exposure to mild adversity in early life (Cohen et al., 1985). Therefore, according to the theory of steeling, we hypothesise that, for a given level of adult SEP, those who had a more socioeconomically disadvantaged childhood will have greater resilience. In other words, we hypothesise that low childhood SEP followed by high adult SEP (i.e. upward intergenerational social mobility) will be associated with greater resilience compared to those who have high SEP in childhood and adulthood.

Key resources that are potentially amenable to change and may promote resilience include lifestyle and social factors (Wister et al., 2016). In particular, leisure time physical activity (LTPA) (Sacker and Cable) and social support (Golden et al., 2009; Kim and Nesselroade, 2003) are related to both physical capability and mental wellbeing. There is evidence that these are socioeconomically patterned (Elhakeem et al., 2015; Turner and Marino, 1994) and may therefore be on the explanatory pathways from SEP to resilience/vulnerability. We hypothesise that those with greater levels of LTPA and social support will have higher levels of resilience and that these resources will partly mediate any relationship between SEP and resilience.

\section{METHODS}

\section{Sample}

The Medical Research Council National Survey of Health and Development (NSHD) is based on a social class stratified sample of 5,362 singleton births on mainland Britain in March 1946 (Wadsworth et al., 2006). Data to operationalise resilience were collected at age 60-64 (Kuh et al., 2011). The 60-64 year data collection 
consisted of a postal questionnaire followed by an invitation to attend an assessment in a clinical research facility or in the study member's home. Of the 3163 people in the target sample, information was obtained from the postal questionnaire and/or visits from 2662 (84.1\%). No attempt was made to contact the remaining 2199 study members: $718(13.4 \%)$ had already died, $594(11.1 \%)$ had previously withdrawn from the study, $567(10.6 \%)$ lived abroad and 320 (5.9\%) had been untraceable for more than ten years (Stafford et al., 2013). Ethical approval for this data collection was obtained from the Greater Manchester Local Research Ethics Committee and the Scotland A Research Ethics Committee.

Resilience

Physical capability

Standardised protocols were used by trained research nurses to assess grip strength, chair rise, standing balance and timed-up-and-go, described elsewhere (Guralnik et al., 2006; Kuh et al., 2005) and summarised below. Grip strength was assessed isometrically using an electronic handgrip dynamometer (Kuh et al., 2006). Three measurements were taken in each hand, with the highest value (in kilograms) recorded used in analyses. Standing balance time was measured by timing how long (up to a maximum of 30 seconds) participants could maintain a one-legged stance with his/her eyes closed. Due to the positive skew of these data, natural logarithm transformation $(\ln ($ seconds+1)) was used to normalise the distribution. Chair rise time was measured as the time taken to rise from a sitting position to a standing position (with straight back and legs) and back to a sitting position 10 complete times. Chair rise speed was calculated as repetitions/minute $((10 /$ time $))$ for use in these analyses. The timed-up-and-go test involved recording the time taken for 
participants to rise from an armless chair, walk 3 meters at a normal pace, turn around, return to the chair, and sit back down(Podsiadlo and Richardson, 1991). The test was performed a single time. Participants were allowed to use walking aids in the timed-up-and-go test $(n=24)$. In instances where participants could not complete the tests for health reasons ( $n=49$ for grip strength, $n=136$ for chair rise, n=89 for standing balance, and n=34 for timed-up-and-go), participants were assigned a value of 0 . As per the method devised by Guralnik, et al, (2006) scores on each measure were rescaled ( 0 (low) to 1 (high)) and summed to create a composite continuous score of physical capability with a range from 0 (unable to perform all 4 tests) to 4 (high performance on all 4 tests). This score has a demonstrated relationship with mortality; further, the use of a composite score demonstrated greater predictive value than any one test (Cooper et al., 2014c).

Mental wellbeing

Prior to the assessment of physical capability, participants self-completed the 14item Warwick-Edinburgh Mental Well-being Scale (WEMWBS) (Tennant et al., 2007) in the postal questionnaire. The WEMWBS was developed to capture both eudemonic and hedonic aspects of mental wellbeing, and includes items on positive affect, satisfying interpersonal relationships, and positive mental functioning (Tennant et al., 2007). Respondents are asked to indicate the frequency, on a fivepoint scale, with which they have experienced each positively worded statement in the last two weeks. Statements include 'I've been feeling good about myself', 'I've been feeling close to other people', 'I've been interested in new things' and 'I've been feeling optimistic about the future'. Higher scores indicate greater wellbeing. Validation studies indicate good construct validity for a single factor structure as well 
as good criterion validity and test-retest reliability, and support the use of the WEMWBS in general population samples (Repetti et al., 2002).

Operationalising resilience

The residual approach to operationalising resilience quantifies the degree to which an individual responds more or less positively to a circumstance than would be predicted by the level of adversity experienced(Miller-Lewis et al., 2013). In the present study, the WEMWBS score quantified a participant's level of wellbeing, and the composite physical capability score quantified the degree of adversity. Resilience was modelled as the difference between observed and predicted WEMWBS score, i.e. residual values, for the physical capability composite score in a linear regression model. The distance between the fitted regression line, i.e. expected WEMWBS score, and the participant's observed WEMWBS score was used as a continuous metric of an individual's level of resilience (Figure 1). The greater the size of the positive residual the greater the resilience the individual demonstrated. Conversely, more negative residual scores indicated greater vulnerability.

\section{Exposures}

Indicators of Socioeconomic Position

Occupation-based social class was coded using the UK Registrar General's Social Class scheme in six categories. Professional, managerial, technical/skilled nonmanual occupations were categorised as non-manual and skilled manual, partly skilled and unskilled as manual occupations. Data for adult head of household social class were collected from the participant at age 53 (or age 43 if missing at $53(n=85)$ and 36 if missing at 53 and $43(n=16))$. Study member's educational attainment by 
age 26 was categorised as "No education or Sub-GCE", "O-Level or equivalent", (typically obtained at age 16) "A-Level or equivalent" (typically obtained at age 18), and "Degree or higher". Indicators of childhood socioeconomic position were based on father's social class, collected in 1950, i.e. participant age 4, and on parental education, collected in 1952, i.e. participant age 6. Father's and mother's educational level was classified as 'primary only', 'primary and further education (no qualifications attained)', 'secondary only or primary and further education or higher', or 'secondary and higher'.

Social Mobility

Intergenerational social mobility was based on childhood and adult social class and classified into four groups (childhood social class/adult social class): manual/manual; manual/non-manual (upward); non-manual/manual (downward); non-manual/nonmanual.

Leisure Time Physical Activity (LTPA)

LTPA was captured at ages 53 and 60-64. In these waves of data collection study members self-reported the number of times they had participated in any sports, vigorous leisure activities or exercises in their spare time, not including getting to and from work, in the past 4 weeks coded as : 0 times (coded 0), 1-4 times (1), or 5 or more times (2). As in previous work (Cooper et al., 2011), a cumulative score was calculated ranging from 0 , i.e. inactive at both ages, to 4 , i.e. LTPA reported 5 or more times at both ages. The validity of these LTPA measures has been considered previously (Cooper et al., 2011) and they have been shown to correlate with monitored levels of daily activity at age 60-64 (Golubic et al., 2014). 


\section{Social Support}

In the social relationships and health literature, studies have typically distinguished structural (capturing the quantity and breadth of a person's social contacts) and functional aspects (capturing the support derived from relationships (Cohen et al., 1985)). Some have also distinguished between positive and negative aspects of functional support. Study members reported the quantity of social contact, perceived social support and marital status based on 13 items at ages 53 and 60-64 (Supplementary table).

To combine social support items to calculate a cumulative score, an indexing procedure was employed, i.e. putting ordinal values on a scale of 0 (lowest) to 10 (highest level of support), for each of the 13 social support variables. Scores from the 13 social support items at age 53 and at age 60-64 were then summed to create a cumulative score ranging from 0 (lowest) to 260 (highest). These scores were then standardised and are reported as z-scores.

Statistical Analysis

Individuals' resilience scores, i.e. residual values from a linear regression of WEMWBS on physical capability, were used as the outcome variable. Residuals were approximately normally distributed indicating a linear association between wellbeing and physical capability. Linear regression models were used to examine the relationship between own and childhood socioeconomic indicators and resilience. The four levels of social mobility were dummy coded, first using downward social mobility as the reference group then using upward social mobility as the reference group to examine differences between different mobility patterns, notably between non-manual/non-manual and upward trajectories. Interactions 
between sex and each socioeconomic indicator were examined but we found no evidence of modification by sex and so sex-adjusted analyses are presented throughout.

Structural equation models, i.e. path analyses, were employed to examine mediation of the associations identified between specific indicators of SEP and resilience through cumulative PA scores and cumulative social support scores. This partitions the total association between SEP and resilience into an indirect association (through the mediators) and a direct association (not explained by included variables). Root means square error approximation (RMSEA), comparative fit index (CFI), and Tucker-Lewis Index (TLI) fit indices were used to assess model fit. Models with RMSEA values lower than 0.05 , CFI values greater than 0.9 , and TLI values greater than 0.95 are deemed to have adequate fit (Hu and Bentler, 1999).

Analytical sample:

A total of 1978 participants provided complete wellbeing data and of these 1756 had complete data on all four physical capability measures, resulting in an analytical sample of 1756 for these analyses. Missing data were handled using full information maximum likelihood, based on the missing at random assumption. Sensitivity analyses were conducted with complete cases as well as with full information maximum likelihood estimation to accommodate for missing data; however, no differences were revealed therefore these results are not presented (data are available on request from the authors).

\section{RESULTS}

Sample 
The sample consisted of $53 \%$ women, with $58 \%$ having educational levels of O-level or lower, and $68 \%$ in non-manual occupations (Table 1 ). Almost $39 \%$ of study members were in the non-manual/non-manual social mobility group and $30 \%$ were in the upward mobility group.

$<$ Table1>

Resilience analysis

There was a positive relationship between higher physical capability and higher WEMWBS scores in a sex-adjusted model (standardised regression coefficient $\beta=2.68, p<0.001,95 \%$ Cl 1.82, 3.54) (Figure 1).

$<$ Figure 1>

There was a trend towards higher resilience in those with higher own and parental educational attainment though these associations did not attain statistical significance (Table 2).

Higher adult social class was associated with higher resilience scores in sexadjusted models (Table 2). Those in the highest social class had resilience scores on average 2.3 units (or 0.29 standard deviations) higher than those in the lowest social class. Childhood social class and parental education were not associated with resilience. Upward and non-manual/non-manual social mobility were associated with greater resilience when compared with downward social mobility; however, when upward social mobility was used as the reference category only an association between upward and downward mobility was observed. Based on this, the mediation analysis focused on explanatory pathways linking adult social class to resilience.

$<$ Table2> 
Higher levels of social support $(\beta=3.27, p<0.001,95 \% \mathrm{Cl} 2.90,3.63)$ and greater participation in LTPA $(\beta=0.58, p<0.001,95 \% \mathrm{Cl} 0.31,0.85)$ were associated with greater resilience-vulnerability scores in sex-adjusted linear regression models. In a sex-adjusted ordinal logistic regression model higher participant social class was associated with higher levels of LTPA (OR=1.36, $p<0.001,95 \% \mathrm{Cl} 1.27,1.44)$. A sex-adjusted linear regression model revealed no association between higher participant social class and social support $(\beta=0.59, p=0.21,95 \% \mathrm{Cl}-0.331 .51)$; therefore, analysis of the mediation of participant social class and resilience by social support was not conducted.

In a mediation model of SEP and resilience by LTPA, the model demonstrated adequate fit $(\mathrm{RMSEA}=0.00(90 \% \mathrm{Cl} 0.00-0.04) ; \mathrm{CFI}=1.00 ; \mathrm{TFI}=1.00)$. The total effect of adult SEP on resilience was $0.46(95 \% \mathrm{Cl} 0.17,0.75)$ and the indirect effect through LTPA was $0.11(95 \% \mathrm{Cl} 0.04,0.17)$, i.e. LTPA accounted for $23.4 \%$ of the variance in the relationship between SEP and resilience.

\section{DISCUSSION}

Adult social class was positively associated with a level of mental wellbeing greater than expected for the level of physical capability observed, i.e. greater resilience (or lower vulnerability). Here we operationalised resilience on a continuum from resilient to vulnerable and found a linear association with adult social class indicating that those in the most advantaged social class were most resilient and those in the most disadvantaged social class were most vulnerable. There was no evidence that this type of resilience in adulthood was associated with childhood SEP, based on father's social class or parental education, or with intergenerational social mobility. 
Since we found no evidence of differences in levels of resilience between the upward and non-manual/non-manual trajectories, these analyses provide no support for the theory of "steeling", i.e. developing resilience through inoculation to adversity and exposure to stress in early life. This theory, has been supported by positive results in animal models (Lyons et al., 2009), but evidence has been much weaker in humans(Rutter, 2012).Our results suggest that current SEP is more closely associated with this type of adult resilience than prior SEP. These findings align with the 'life course model of multimorbidity resilience theory', in which access to and accumulation of resources is suggested to foster greater resilience (Wister et al., 2016). Regardless of their childhood SEP, individuals with high adult SEP were more likely to demonstrate greater resilience. Accumulation of resources is more closely associated with resilience than perseverance in the absence of resources.

Our findings supported our hypothesis that individuals who had greater LTPA, and social support would demonstrate greater levels of resilience and lower levels of vulnerability. In addition, the cumulative scores of LTPA and social support at age 53 and 60-64 were associated with greater resilience, supporting the accumulation of resources component of the 'life course model of multimorbidity resilience' (Wister et al., 2016). Whilst a relationship was observed between higher LTPA and higher adult social class, no relationship was observed for our composite measure of structural and functional social support and social class leading us to conclude that social support is not on the explanatory pathway between adult social class and resilience in this setting. LTPA, however, was shown to mediate part of the relationship between SEP and resilience. These results suggest that socioeconomic inequalities in resilience in terms of maintaining wellbeing given low levels of physical capability may be reduced through the fostering of health behaviours, such 
as LTPA. However, the relationship between LTPA and physical capability is likely bidirectional. To some extent, those with greater physical capability may be more able to be physically active. We are unable to tell from these analyses whether increasing LTPA would lead to greater resilience or whether greater participation in LTPA is a marker of greater physical capability which is not captured by the performance measures we have considered.

These results must be interpreted with the following limitations in mind. As with any longitudinal study, attrition via death and dropout may introduce bias and/or compromise the generalisability of the findings. To address missing covariate and exposure data, full information maximum likelihood estimation (FIML) was conducted in linear regression models under a missing at random assumption. However, we did not include those missing data on physical capability and wellbeing. We know from earlier work that non-responders at age 60-64 had lower lifetime SEP and had lower physical capability at the previous sweep (age 53), though they did not differ from responders in mental health (Stafford et al., 2013). We have examined LTPA and social support as mediators of SEP and resilience; however, many factors beyond these variables, e.g. diet and smoking status, may also have had an impact on individuals' resilience-vulnerability. LTPA is just one domain of physical activity that is socioeconomically patterned (Elhakeem et al., 2015), other domains, e.g. active transport and occupational activity, were not included in these analyses; further investigations are required to examine physical activity beyond LTPA. Participants in the present study were aged 60-64; therefore, findings from this study may not be generalisable to other age groups. Additionally, we have examined only one form of resilience, i.e. the relationship between physical capability and mental wellbeing. There are many other forms of resilience that use different adversity and outcome 
dyads, to which the findings from the present study may not be generalisable.

Further investigation is required to examine the relationship between resources in other resilience operationalisations.

The MRC National Survey of Health and Development is a socially stratified population-representative study of ageing, providing longitudinal data on resilience, socioeconomic indicators, and sociobehavioral measures. A strength of these analyses is the prospective collection of data from birth to age $60-64$; recall bias is, therefore, minimised. Through the examination of mediators that are the potentially modifiable, there is opportunity for intervention; for example, LTPA is a form of physical activity that is amenable to change.

The current study suggests that modifiable aspects of lifestyle, i.e. LTPA, may play a role in the mediation of socioeconomic (dis)advantage in the fostering of resilience. Age-related declines in physical capability have been associated with increased risk of disability (Manton et al., 1997; Wister et al., 2016). However, whilst some "vulnerable" individuals experience low wellbeing when facing limited capability undertaking the physical tasks of daily living, other "resilient" individuals have relatively high wellbeing for their level of physical capability. Socioeconomic advantage, LTPA, and social support are positively correlated with this resilience. Lower SEP is associated with poorer physical capability(Cooper et al., 2014a; Cooper et al., 2014b; Pierce et al., 2012) but these findings provide some preliminary evidence that interventions to raise LTPA levels among those who are more socioeconomically disadvantaged may help promote wellbeing in the face of this kind of adversity. Through individual- and policy-level interventions to increase LTPA, it may be possible to offset the negative implications of lower SEP, which has the 
potential to facilitate more positive ageing trajectories and greater wellbeing across the life course.

1

2

3

4

5

6

7

8

9

10

11

12

13

14

15

16

17

18

19

20

21

22

23

24

25

26

27

28

29

30

31

32

33

34

35

36

37

38

39

40

41

42

43

44

45

46

47

48

49

50

51

52

53

54

55

56

57

58

59

60
19

https://mc.manuscriptcentral.com/ipg 


\section{Acknowledgements}

The authors thank the study participants for their continuing participation in the MRC NSHD. They also thank members of the NSHD scientific and data collection teams who have been involved in the NSHD data collections.

This study and RC, DK and MS are funded by the UK MRC (programme codes MC_UU_12019/1; MC_UU_12019/4; MC_UU_12019/5).

TDC is supported by a Canadian Institutes of Health Research Postdoctoral Fellowship (MFE-146676).

Conflict of Interest:

All authors declare no conflict of interest.

Author contributions:

TDC, MS, RC, and DK conceived of the study. TDC conducted the analysis and wrote the first draft. MS, RC, and DK aided in the analysis and interpretation of the results. All authors contributed to editing and revising the manuscript. 


\title{
References
}

\begin{abstract}
Albrecht, G. L. and Devlieger, P. J. (1999). The disability paradox: high quality of life against all odds. Social Science \& Medicine, 48, 977-988.

Antonovsky, A. (1996). The salutogenic model as a theory to guide health promotion Health Promotion International, 11, 11-18.

Cohen, S., Mermelstein, R., Kamarck, T. and Hoberman, H. M. (1985). Measuring the Functional Components of Social Support. In I. G. Sarason and B. R. Sarason (Eds.) Social Support: Theory, Research and Applications (pp. 73-94) Dordrecht: Springer Netherlands.

Cooper, R., Hardy, R., Sayer, A. A. and Kuh, D. (2014a). A life course approach to physical capability In D. Kuh, R. Cooper, R. Hardy, M. Richards and Y. Ben-Shlomo (Eds.) A life course approach to healthy ageing (pp. 16-31) Oxford, UK: Oxford University Press.
\end{abstract}

Cooper, R., Mishra, G. D. and Kuh, D. (2011). Physical activity across adulthood and physical performance in midlife: findings from a British birth cohort. Am J Prev Med, 41, 376-384.

Cooper, R., et al. (2014b). Physical capability and subsequent positive mental wellbeing in older people: findings from five HALCyon cohorts. Age (Dordr), 36, 445456.

Cooper, R., Strand, B. H., Hardy, R., Patel, K. V. and Kuh, D. (2014c). Physical capability in mid-life and survival over 13 years of follow-up: British birth cohort study. BMJ, 348, g2219. 
Elhakeem, A., Cooper, R., Bann, D. and Hardy, R. (2015). Childhood

socioeconomic position and adult leisure-time physical activity: A systematic review. International Journal of Behavioral Nutrition and Physical Activity, 12.

Golden, J., et al. (2009). Loneliness, social support networks, mood and wellbeing in community-dwelling elderly. International Journal of Geriatric Psychiatry, 24, 694700.

Golubic, R., et al. (2014). Levels of physical activity among a nationally representative sample of people in early old age: results of objective and selfreported assessments. Int J Behav Nutr Phys Act, 11, 58.

Guralnik, J. M., Butterworth, S., Wadsworth, M. E. and Kuh, D. (2006). Childhood socioeconomic status predicts physical functioning a half century later. Journals of Gerontology - Series A Biological Sciences and Medical Sciences, 61, 694-701. Hu, L. T. and Bentler, P. M. (1999). Cutoff criteria for fit indexes in covariance structure analysis: Conventional criteria versus new alternatives. Structural Equation Modeling, 6, 1-55.

Kim, J. E. and Nesselroade, J. R. (2003). Relationships among social support, selfconcept, and wellbeing of older adults: A study of process using dynamic factor models. International Journal of Behavioral Development, 27, 49-65.

Kuh, D., Bassey, E. J., Butterworth, S., Hardy, R. and Wadsworth, M. E. (2005). Grip strength, postural control, and functional leg power in a representative cohort of British men and women: associations with physical activity, health status, and socioeconomic conditions. Journals of Gerontology - Series A Biological Sciences and Medical Sciences, 60, 224-231. 
Kuh, D., et al. (2006). Developmental origins of midlife grip strength: Findings from a birth cohort study. Journals of Gerontology - Series A Biological Sciences and Medical Sciences, 61, 702-706.

Kuh, D., et al. (2011). Cohort profile: updating the cohort profile for the MRC National Survey of Health and Development: a new clinic-based data collection for ageing research. International Journal of Epidemiology, 40, e1-9.

Lyons, D. M., Parker, K. J., Katz, M. and Schatzberg, A. F. (2009). Developmental cascades linking stress inoculation, arousal regulation, and resilience. Frontiers in Behavioral Neuroscience, 3, 6.

Manton, K. G., Corder, L. and Stallard, E. (1997). Chronic disability trends in elderly United States populations: 1982- 1994. Proceedings of the National Academy of Sciences of the United States of America, 94, 2593-2598.

Marmot, M. (2005). Social determinants of health inequalities. Lancet, 365, 10991104.

Masten, A. S. (2001). Ordinary magic: Resilience processes in development. American Psychologist, 56, 227-238.

Miller-Lewis, L. R., Searle, A. K., Sawyer, M. G., Baghurst, P. A. and Hedley, D. (2013). Resource factors for mental health resilience in early childhood: An analysis with multiple methodologies. Child and adolescent psychiatry and mental health, 7, 6-6.

Ong, A. D., Bergeman, C. S., Bisconti, T. L. and Wallace, K. A. (2006).

Psychological resilience, positive emotions, and successful adaptation to stress in later life. Journal of personality and social psychology, 91, 730-749. 
Pierce, M. B., Kuh, D. and Hardy, R. (2012). The role of BMI across the life course in the relationship between age at menarche and diabetes, in a British Birth Cohort. Diabetes Medicine, 29, 600-603.

Podsiadlo, D. and Richardson, S. (1991). The timed 'Up and Go': A test of basic functional mobility for frail elderly persons. Journal of the American Geriatrics Society, 39, 142-148.

Repetti, R. L., Taylor, S. E. and Seeman, T. E. (2002). Risky families: Family social environments and the mental and physical health of offspring. Psychological Bulletin, $128,330-366$.

Rutter, M. (2012). Resilience as a dynamic concept. Development and Psychopathology, 24, 335-344.

Sacker, A. and Cable, N. (2006). Do adolescent leisure-time physical activities foster health and well-being in adulthood? Evidence from two British birth cohorts. European Journal of Public Health, 16, 331-335.

Stafford, M., et al. (2013). Using a birth cohort to study ageing: representativeness and response rates in the National Survey of Health and Development. European Journal of Ageing, 10, 145-157.

Tennant, R., et al. (2007). The Warwick-Edinburgh Mental Well-being Scale (WEMWBS): development and UK validation. Health and Quality of Life Outcomes, $5,63$.

Turner, R. J. and Marino, F. (1994). Social support and social structure: a descriptive epidemiology. Journal of Health and Social Behavior, 35, 193-212.

Wadsworth, M., Kuh, D., Richards, M. and Hardy, R. (2006). Cohort Profile: The 1946 National Birth Cohort (MRC National Survey of Health and Development). International Journal of Epidemiology, 35, 49-54. 
Windle, G., Woods, R. T. and Markland, D. A. (2010). Living with III-Health in Older Age: The Role of a Resilient Personality. Journal of Happiness Studies, 11, 763-777. Wister, A. V., Coatta, K. L., Schuurman, N., Lear, S. A., Rosin, M. and MacKey, D. (2016). A Lifecourse Model of Multimorbidity Resilience: Theoretical and Research Developments. International Journal of Aging \& Human Development, 82, 290-313. 


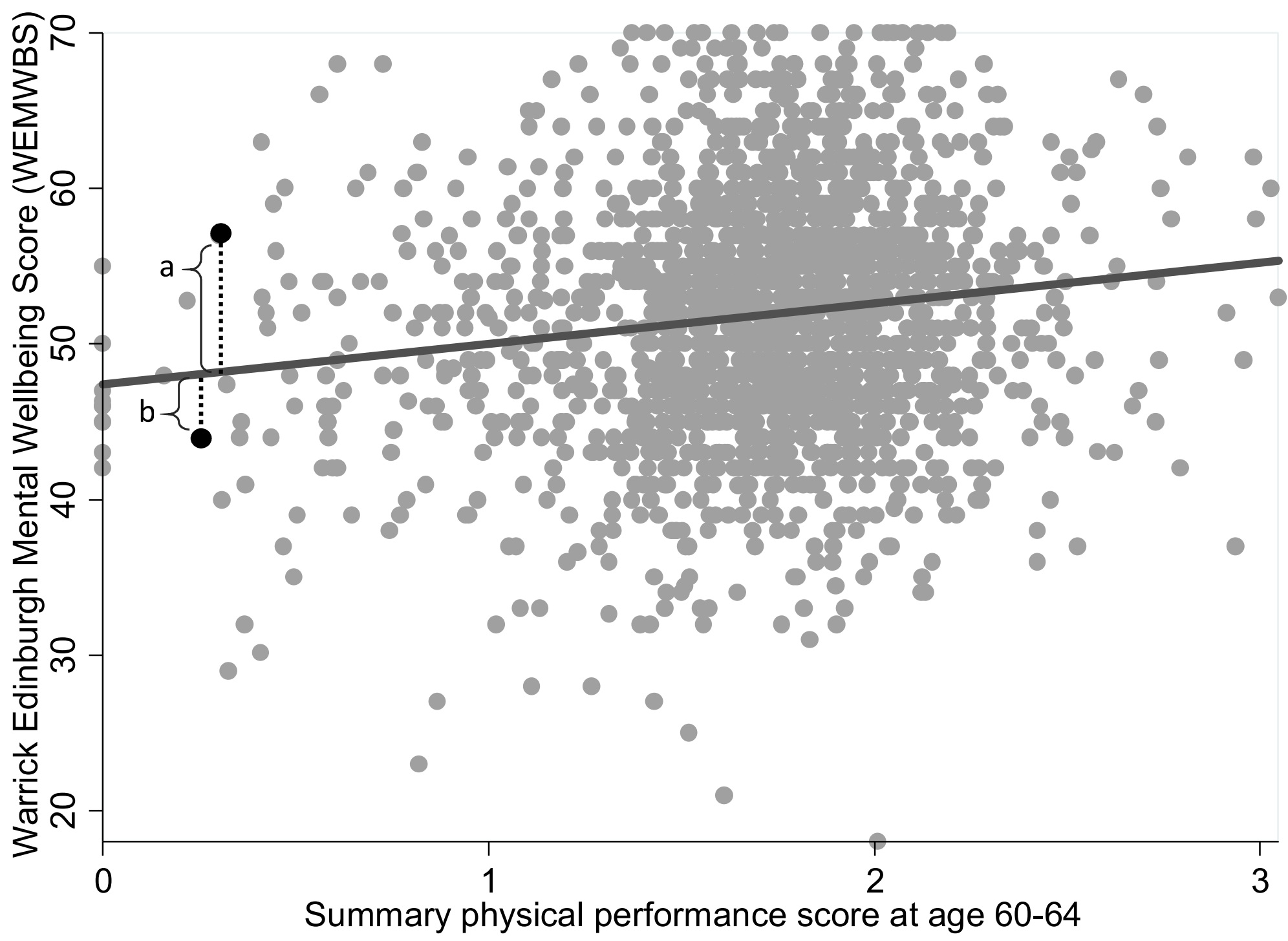

Figure 1: Wellbeing linearly regressed on summary objective physical performance at age 60-64 (standardised regression coefficient $\beta=2.68, p<0.001,95 \% \mathrm{Cl} 1.82,3.54$ ).

a Positive residual value indicating greater observed wellbeing score than would be expected by the fitted regression line;

${ }^{\mathrm{b}}$ Negative residual value indicating lower observed wellbeing score than would be expected by the fitted regression line 
Table 1 Characteristics of the MRC NSHD (sample restricted to those with complete data on wellbeing and physical capability at age 60-64)

\begin{tabular}{|c|c|c|c|c|}
\hline & & & $\mathrm{n}$ & Mean(SD) / \% \\
\hline Warwick-Edinburs & Mental Well & ing Score & 1756 & $51.8(8.0)$ \\
\hline Composite Physic & I Capability S & ore & 1756 & $1.7(0.4)$ \\
\hline & & Men & 822 & $46.8 \%$ \\
\hline & & Women & 934 & $53.2 \%$ \\
\hline & & None/Sub-GCE & 601 & $36.3 \%$ \\
\hline & Fducation & O-level & 351 & $21.2 \%$ \\
\hline & cuucation & A level or equivalent & 502 & $30.3 \%$ \\
\hline & & Degree or higher & 203 & $12.3 \%$ \\
\hline Adulthood & & V Unskilled & 50 & $2.9 \%$ \\
\hline & & IV Partly skilled & 129 & $7.4 \%$ \\
\hline & Social loca & IIM Skilled (Manual) & 371 & $21.4 \%$ \\
\hline & social class & IIINM Skilled (Non-manual) & 224 & $12.9 \%$ \\
\hline & & II Intermediate & 747 & $43.0 \%$ \\
\hline & & I Professional & 215 & $12.4 \%$ \\
\hline & & V Unskilled & 77 & $4.8 \%$ \\
\hline & & IV Partly skilled & 300 & $18.6 \%$ \\
\hline & Social class & IIM Skilled (Manual) & 458 & $28.4 \%$ \\
\hline & & IIINM Skilled (Non-manual) & 352 & $21.8 \%$ \\
\hline & & II Intermediate & 297 & $18.4 \%$ \\
\hline & & I Professional & 128 & $7.9 \%$ \\
\hline & & $\begin{array}{l}\text { Primary only } \\
\text { Primary and further education }\end{array}$ & 796 & $51.5 \%$ \\
\hline Childhood & Father's & (no qualifications) & 208 & $13.5 \%$ \\
\hline & & $\begin{array}{l}\text { Secondary only or primary and } \\
\text { further education }\end{array}$ & 242 & $15.7 \%$ \\
\hline & & Secondary and higher & 299 & $19.4 \%$ \\
\hline & & Primary only & 870 & $55.7 \%$ \\
\hline & Mother's & $\begin{array}{l}\text { Primary and further education } \\
\text { (no qualifications) }\end{array}$ & 245 & $15.7 \%$ \\
\hline & Education & Secondary only or primary and & & \\
\hline & & further education & 212 & $13.6 \%$ \\
\hline & & Secondary and higher & 235 & $15.0 \%$ \\
\hline & & Downward & 148 & $9.3 \%$ \\
\hline Intergenerational & Social class & Manual/Manual & 348 & $21.8 \%$ \\
\hline Social Mobility & & Non-manual/Non-manual & 621 & $38.9 \%$ \\
\hline & & Upward & 478 & $30.0 \%$ \\
\hline & & None & 709 & $42.6 \%$ \\
\hline Leisure Time & 1999 & 1-4 times & 340 & $20.4 \%)$ \\
\hline Physical Activity & & $\geq 5$ times & 614 & $36.9 \%)$ \\
\hline (in last 4 weeks) & ?009 & None & 1093 & $63.1 \%$ \\
\hline & $\angle U U=$ & 1-4 times & 241 & $13.9 \%$ \\
\hline
\end{tabular}




\begin{tabular}{rrrrr} 
& & $\geq 5$ times & 398 & $23.0 \%$ \\
\hline \multirow{3}{*}{ Marital Status } & Married & 1347 & $81.0 \%$ \\
\cline { 2 - 4 } & \multirow{2}{*}{1999} & Not-married & 316 & $19.0 \%$ \\
\cline { 2 - 5 } & \multirow{2}{*}{2009} & Married & 1246 & $79.0 \%$ \\
& \multirow{2}{*}{} & Not-married & 331 & $21.0 \%$
\end{tabular}


Table 2: Resilience linearly regressed on socioeconomic indicators in sexadjusted models

\begin{tabular}{|c|c|c|c|c|}
\hline & \multirow[b]{2}{*}{ Education $^{a}$} & \multirow{2}{*}{$\frac{\beta}{0.28}$} & \multicolumn{2}{|c|}{$\begin{array}{c}95 \% \\
\text { Confidence } \\
\text { Interval }\end{array}$} \\
\hline \multirow{2}{*}{$\begin{array}{l}\text { Adult } \\
\text { Socioeconomic } \\
\text { Position }\end{array}$} & & & -0.52 & 0.97 \\
\hline & Social Class $^{b}$ & 0.46 & 0.17 & 0.75 \\
\hline \multirow{3}{*}{$\begin{array}{l}\text { Childhood } \\
\text { Socioeconomic } \\
\text { Position }\end{array}$} & Father's Education $^{c}$ & -0.06 & -0.39 & 0.26 \\
\hline & Mother's Education $^{c}$ & -0.16 & -0.50 & 0.19 \\
\hline & Father's Social Class ${ }^{\mathrm{b}}$ & 0.01 & -0.28 & 0.31 \\
\hline \multirow{4}{*}{ Social Mobility } & Downward & Referent & & \\
\hline & Manual/Manual & 1.20 & -0.31 & 2.72 \\
\hline & Non-manual/Non-manual & 1.81 & 0.40 & 3.22 \\
\hline & Upward & 1.99 & 0.54 & 3.44 \\
\hline Physical Activity & Cumulative Score $^{d}$ & 0.58 & 0.31 & 0.85 \\
\hline Social Support & Cumulative Score ${ }^{\mathrm{e}}$ & 3.27 & 2.90 & 3.63 \\
\hline
\end{tabular}

Regression coefficients represent the standardised difference in mean residual scores per 1 category/unit change in specified socioeconomic indicator

a Categorised as: 0 "No education or Sub-GCE"; 1 "O-Level or equivalent" (typically obtained at age 16); 2 "A-Level or equivalent" (typically obtained at age 18); 3 "Degree or higher"

b Categorised as: 0 "Unskilled"; 1 "Partly skilled"; 2 "Skilled (Manual)"; 3 "Skilled (Non-manual)"; 4 "Intermediate"; 5 "Professional"

c Categorised as: 0 "Primary only"; 1 "Primary and further education (no qualifications attained)"; 2 "Secondary only or primary and further education or higher"; 3 "Secondary and higher"

${ }^{d}$ Higher scores indicate greater physical activity

${ }^{\mathrm{e}}$ Higher scores indicate greater social support 